\title{
Rare Bilateral Caudate Infarction in a Patient with a Common Circle of Willis Variant
}

\author{
Mimma G. Anello, Timothy L. Miao, Sachin K. Pandey, Jennifer L. Mandzia
}

Keywords: Stroke, Stroke imaging, Behavioral neurology, Neuroanatomy

doi:10.1017/cjn.2019.233

Can J Neurol Sci. 2019; 46: 593-594

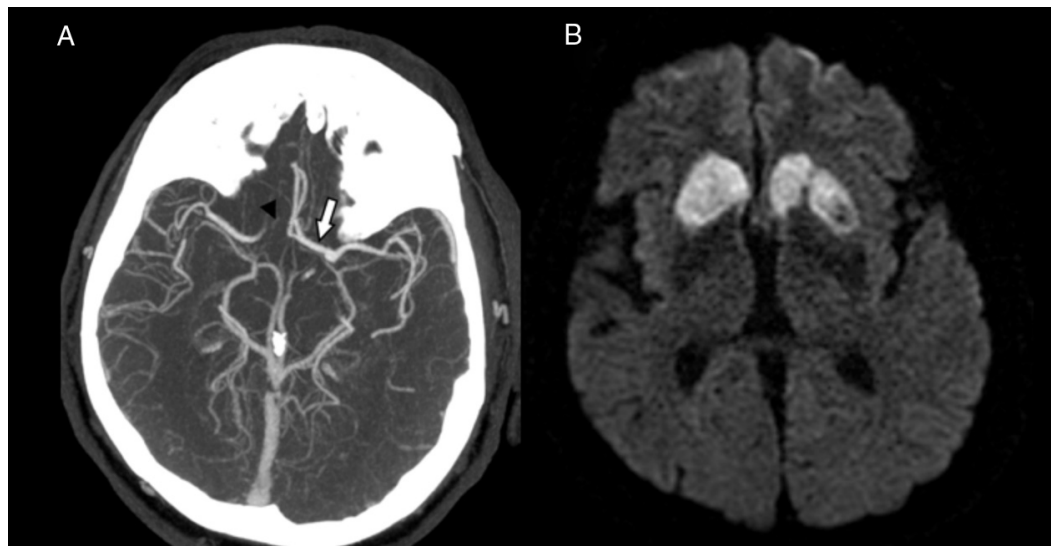

Figure 1: (A) Axial image from the maximum intensity projection of the CT angiogram demonstrates the left internal carotid artery supplying both anterior cerebral arteries via a large Al segment (white arrow) with absence of the right Al segment (black arrowhead). (B) Axial image from the diffusion-weighted sequence MRI demonstrates bilateral caudate and putamen infarcts.

A 62-year-old male presented to hospital with acute aphasia. His past medical history was significant for a previous left middle cerebral artery stroke, from which he fully recovered, hypertension, dyslipidemia, coronary artery disease, one episode of atrial fibrillation postoperatively, and thalidomide exposure in utero. Although initially he was thought to be aphasic, on further examination, he demonstrated significant abulia. His level of consciousness was normal, and neurological examination was otherwise unremarkable. A CT angiogram of the head and neck was performed. The patient was not a candidate for acute therapy, as he had established stroke on imaging, and the time of onset was unclear.

The CT angiogram demonstrated a left internal carotid artery (ICA) supplying both anterior cerebral arteries (ACAs) with absence of the right A1 segment (Figure 1A). The MRI demonstrated symmetric bilateral caudate and putamen infarctions (Figure 1B).

During his admission, he went into rapid atrial fibrillation, and the etiology of his stroke was felt to be cardioembolic. Clinically, he remained apathetic and did not show concern over his condition. His abulia with regard to speech and spontaneous movement improved during his stay in hospital. His most prominent finding on neurological examination was a delay in initiation of voluntary movements.

Variant anatomy of the circle of Willis, although common, may cause atypical stroke presentations and unique imaging findings. Within the circle of Willis, normal anatomy consists of two ACAs joined together by an anterior communicating artery (AComA). Autopsy studies have shown abnormalities of the circle of Willis to be common, occurring in more than $50 \%$ of brains studied. In these post-mortem examinations, absence of a proximal ACA was seen in $0.4 \%$ of cases. Absence of a proximal

From the Department of Clinical Neurosciences, London Health Sciences Centre, Western University, London, Ontario, Canada (MGA, JLM); Department of Medical Imaging, London Health Sciences Centre, University Hospital, London, Ontario, Canada (TLM); Department of Medical Imaging, Western University - Schulich School of Medicine, London, Ontario, Canada (SKP).

Received April 10, 2019. Final Revisions Submitted June 6, 2019. Date of AcCePtance June 10, 2019.

Correspondence to: Mimma G. Anello, Department of Clinical Neurosciences, London Health Sciences Centre, Western University, London, Ontario, N6A 5A5 Canada. Email: mimma.anello@1hsc.on.ca 
ACA is associated with a smaller ipsilateral ICA and larger contralateral ACA that divides into a left and right branch. ${ }^{1}$ This variant increases the potential ischemic stroke volume in the ACA territory of the frontal lobes during ischemic events. ${ }^{2}$ In our case, left ACA occlusion secondary to a cardioembolic mechanism resulted in bilateral ACA territory infarctions due to the division of the left ACA into a left and right branch.

Bilateral caudate infarction is rare, and its association with an absence of A1 segment has only been previously described twice. ${ }^{3}$ In a study looking at 240 patients with lesions in the caudate, putamen, or globus pallidus, the most prominent behavioral symptom in those with caudate lesions was abulia. ${ }^{4}$ This was observed in our patient who demonstrated apathy with loss of spontaneous speech and delayed voluntary movement.

In conclusion, although variations in the configuration of the circle of Willis are common, atypical and rare stroke presentations can occur from ischemia when variations in anatomy exist. This is only the third report of bilateral caudate infarction secondary to this normal variant anatomy of the circle of Willis.

\section{ACKNOWLedGements}

We acknowledge all the nurses, speech language pathologists, physiotherapists, occupational therapists, staff physicians, and resident physicians who helped take care of our patient and ensure that he was able to return home.

\section{Disclosures}

No disclosures relevant to the manuscript.

\section{Statement of Authorship}

MGA contributed to the case, literature review, drafting, and revising the manuscript. TLM contributed to the imaging, imaging description, and literature review. SKP contributed to the imaging and imaging description. JLM contributed to the case and revising the manuscript.

\section{REFERENCES}

1. Kapoor K, Singh B, Dewan L. Variations in the configuration of the circle of Willis. Anat Sci Int. 2008;83(2):96-106.

2. Markowicz G, Poniatowska R, Lusawa M. Variants of cerebral arteries - anterior circulation. Pol J Radiol. 2013;78(3):42-7.

3. Fukuoka T1, Osawa A, Ohe Y, Deguchi I, Maeshima S, Tanahashi N. Bilateral caudate nucleus infarction associated with a missing A1 segment. J Stroke Cerebrovasc Dis. 2012;21(8):908.e11-e12.

4. Bhatia K, Marsden C. The behavioural and motor consequences of focal lesions of the basal ganglia in man. Brain. 1994;117(4): $859-76$. 